\title{
Trend forecasting of main groups of causes-of-death in Iran using the Lee-Carter model
}

\author{
Katayoun Jahangiri ${ }^{1}$, Saeide Aghamohamadi*2, Ardeshir Khosravi ${ }^{2}$, Elaheh Kazemi ${ }^{2}$ \\ Received: 10 Feb 2018 \\ Published: 12 Dec 2018
}

\section{Abstract}

Background: Authoritative information and description of the causes of mortality trends is one of the most basic principles of evaluation of the health sector in all countries. Therefore, this study aims to predict the trend in main groups of causes of death in the Islamic Republic of Iran.

Methods: The study population comprised all deaths recorded in the system of registration and classification of causes of death of Ministry of Health and Medical Education during the years 2006 to 2015. The information was collected via death certificate, burial permit, and information forms. In order to forecast the trends of causes-of-death, Lee Carter model was employed in a demographic package 18.1 of $\mathrm{R}$ software version 3.3.1.

Results: Based on the results, the crude death rate in the total population has reduced from 435.86 in 2006 to 405.01 per 100000 population in 2035. It is anticipated that endocrine, nutritional and metabolic diseases with the rate of 197.71 per 100000 population will be responsible for the highest causes of death in the year 2035 and from 2021 onwards, the mental and behavioral disorders will be responsible for the lowest rate of mortality.

Conclusion: Non-communicable diseases have a major role in the deaths of Iranian population; therefore, to reduce the impact of non-communicable diseases on individuals and society, a holistic approach is needed which requires all sectors to cooperate.

Keywords: Prediction, Causes of death, Iran, Lee Carter

Conflicts of Interest: None declared

Funding: None

*This work has been published under CC BY-NC-SA 1.0 license.

Copyright $\odot$ Iran University of Medical Sciences

Cite this article as: Jahangiri K, Aghamohamadi S, Khosravi A, Kazemi E. Trend forecasting of main groups of causes-of-death in Iran using the Lee-Carter model. Med J Islam Repub Iran. 2018 (12 Dec);32:124. https://doi.org/10.14196/mjiri.32.124

\section{Introduction}

Credible information on the causes of death and mortality trends and its changes is one of the most basic principles of planning, management, and evaluation of the health sectors in all countries. Collection and analysis of information on causes of death have been utilized for several years as an instrument for assessment and monitoring of community health promotion and to determine priorities of health measures (1).

International Statistical Classification of Diseases and Related Health Problems (ICD) and its rules are the standard and basis for determining the exact cause of death that helps countries better identify the pattern of death. In fact, ICD is a diagnostic classification and an international

\footnotetext{
Corresponding author: Dr Saeide Aghamohamadi, Aghamohamadi@health.gov.ir

1. Department of Health in Disasters and Emergency, School of Health, Safety, Environment (HSE), Shahid Beheshti University of Medical Sciences, Tehran, Iran

2. Deputy of Health, Ministry of Health and Medical Education, Tehran, Iran
}

standard for all public and epidemiologic purposes as well as for the number of objectives of health care services (2). Thus using this classification has considerably helped to recognize, monitor and predict trends and causes of death and led to the determination of health priorities, the allocation of resources and priorities for "health-oriented development" of the health sector (3).

Moreover, analysis of trends in cause of death based on ICD is of great importance in anticipating overall death rates. The need for better methods for predicting mortality trends and the impact of various factors on the epidemiology of death has been recognized for some organizations (4). As the importance of predicting mortality has an

\section{$\uparrow$ What is "already known” in this topic:}

World Health Organization anticipated that diseases of the circulatory systems, neoplasms and diseases of the respiratory will be the three major causes of death in the world in 2030 .

\section{$\rightarrow$ What this article adds:}

In Iran until the year 2035 anticipated, endocrine, nutritional and metabolic diseases, diseases of the circulatory system and neoplasms will be the three major causes of death. 
increasing trend, several methods have also been developed for modeling and forecasting mortality (5).

Most new methods for predicting mortality employ twofactor models with two factors of age and time period. Lee Carter model is one of these two-factor models for predicting mortality (6). According to the study by Maria Chiara that examines the different approaches of predicting causesof death, the Lee Carter model is a valid method for predicting the cause of death with a linear trend which is superior to other simplifying assumptions that are dependent on subjective judgment (7).

Moreover, this model can cover most of the changes in mortality rates in developing countries' population (8) and based on the results of the study by Komijani, Lee Carter model has high power and efficiency to predict mortality in Iran (9). So this study aims to predict the crude and the main groups of causes of death in Iran until 2035 using Lee Carter model.

\section{Methods}

In this descriptive and analytical study, the study population included all deaths recorded in the system of registration and classification of causes of death in the Ministry of Health and Medical Education of Iran during the years 2006 to 2015 . The classification was based on the cause of death, age groups, and gender. The data were collected from different sources such as hospitals, clinics, physicians' offices, Forensic Medicine Organization, health centers, and cemeteries. Data collection tools were death certificate forms, burial permit, and information forms.

After data collection, causes of death were determined, coded and recorded by the coders based on the International Statistical Classification of Diseases (2) and then the recorded items were evaluated in terms of quality. In order to enhance the quality of the data, the codes of impossible causes of death in terms of gender and age, codes of improbable causes of death in terms of fatality of causes, codes of causes of death from ill-defined or null status by World Health Organization Standard Guideline were corrected (10).

Furthermore, the number of deaths in terms of causes of death and gender and age groups were calculated, the rate of death was computed by dividing the number of causes of death to the population of that age group/gender per 100000 population.

It should be noted that for the denominator of the rates, population estimates between two censuses of Statistical Center of Iran were employed for the years 2007-2010, 2012-2015 and for the years 2006 and 2011 the census information of that year was used (11).

The Lee Carter model was used to predict death rates. Lee-Carter model as a method of extrapolation is a combination of a rich demographic model and time series method. Generally, one of the strengths of Lee Carter methods and other extrapolation methods is their application in a situation where there is a linear trend of mortality rates in the age groups (12). Considering the existence and calculation of the linear trend of crude and cause of death in this study, this method was utilized.
Model Structure

Crude death rate at age $x$ and time $t$ in a society is shown with $M_{(x, t)}$ and is calculated using this relationship:

$$
\begin{gathered}
M_{(x, t)}=\frac{d_{x, t}}{L_{x, t}}, t=t_{1},\left(t_{1}+1\right), \ldots,\left(t_{1}+T-1\right), x \\
=x_{1}, x_{2}, \ldots, x_{N}
\end{gathered}
$$

Where $d_{x, t}$ and $L_{x, t}$ indicate the number of dead people and the population at risk of death at age $x$ and time $t$ for the given society, $t_{1}$ shows the first time point and $N$ is the number of age groups under study.

The structure of the proposed model of Lee-Carter is presented as follows:

$$
\operatorname{Lnm}_{(x, t)}=a_{x}+b_{x} k_{t}+\varepsilon_{x, t}
$$

Where $\operatorname{Lnm}_{(x, t)}$ indicates the natural logarithm of death rates observed in the age $x$ and in year $t$ and $a_{x}, b_{x}$ and $k_{t}$ are age and time dependent parameters, respectively. $a_{x}$ indicates the average time logarithm of death rates at age $x$, in other words, $\varepsilon_{x, t}$ illustrates the overall shape of the mortality rate's curve; $k_{t}$ is the mortality index in year $t$ which indicates the main trend in the natural logarithm of mortality rates for all ages over time; and $b_{x}$ shows the rate of changes in the mortality rate logarithm at age $x$ to (per) changes in the mortality index over time. Component, $\varepsilon_{x, t}$ is equal to the component error at age $x$ and time $t$. According to equation (2), the following relation is obtained:

$$
\frac{d}{d t} \operatorname{Lnm}_{(x, t)}=b_{x}\left(\frac{d}{d t} k_{t}\right)
$$

According to equation (3), if the $k_{t}$ mortality index decreases linearly over time, it remains constant and age specific mortality rate will decrease by its constant exponential rate. The error terms, $\varepsilon_{x, t}$, have a Gaussian distribution with zero mean and variance $\sigma 2 \varepsilon$ and represents part of the changes in age specific mortality rate that cannot be explained by the model. Lee and Carter believe that $k_{t}$ parameter covers the major scatter in the data and, as a result, the variance of the error term is constant over time (13).

\section{Predicting Mortality Rate}

After estimating the parameters of the model and agespecific mortality rates, the Lee-Carter model is employed to predict the mortality rate. For this purpose, Lee and Carter first developed time series model for $k_{t}$ and then by predicting the number of $k_{t}$, they predicted mortality rate $m_{(x, t)}$ for each age group, and at any particular time. For the prediction, first $k_{t}$ is predicted applying time series modeling and its future values. Finding the best model for $k_{t}$ is very important because an inappropriate model will result in incorrect prediction of the future behavior of mortality rate. Random Walk model with Drift for $k_{t}$ is presented as follows:

(4)

$$
k_{t}=k_{t-1}+\theta+\varepsilon_{t}, \quad \varepsilon_{t} \sim N\left(0, \sigma_{r w}^{2}\right)
$$

In the second stage of prediction, values for age-specific mortality rates are anticipated. Regardless of the errors 
term, changes in the rate of deaths in a particular year are completely dependent together and are linear functions of time variable parameter of $k_{t}$. Therefore, to calculate confidence intervals of mortality rates in any age group and in any given year, only $k_{t}$ confidence interval should be calculated. Prediction of mortality rates with respect to the estimated parameter values and predicted values of is calculated according to the following equation (13):

(5)

$m_{(x, t+s)}^{\wedge}=m_{(x, t)} \exp \left(b_{x}^{\wedge}\left(k_{t+s}^{\wedge}-k_{t}^{\wedge}\right)\right), s=1,2, \ldots, S$

In this study for predicting crude rates and the main groups' rate of causes of death in Iran, after calculating the rate of crude and cause-specific death in 5-year age groups during the study, the 20 -year trend was predicted applying the Lee Carter model.

It should be noted that, in this model, the International Classification of Diseases was used to predict the trend of the causes of death. Of the total 21 main disease groups in this classification, the diseases that cannot be considered as the underlying causes of death and disease groups with negligible or zero value in age and gender groups were excluded from the study. Finally, the forecast for 13 major groups of causes of death was conducted.

\section{Data analysis tools}

Demography package (18.1) of Lee Carter model in the $\mathrm{R}$ software version 3.3.1 was utilized to predict mortality rates.

\section{Ethics}

This study is not based on individual information and medical intervention and use of mortality data of the Islamic Republic of Iran, formal permission of Iran's Ministry of Health and Medical Education with latter number D308/22604 have been issued.

\section{Results}

In this study, crude death rates during the years 2006 to 2015 in the 5-year age groups and in total population were calculated and then the rates forecasted for the next 20 years utilizing the Lee Carter model, as shown in Table 1.

According to the results, the crude death rate in all age groups under 75 years from 2006 to 2015 had a decreasing trend, and this negative trend is expected to continue until 2035. Furthermore, in age groups over 75 years, this trend has been increasing.

After calculating the crude death rate, the causes of death rates were forecasted until the year 2035 (Table 2).

According to the results of the forecast for 2035, disease groups such as intentional and unintentional injuries, congenital malformations, deformities and chromosomal abnormalities, diseases of the circulatory systems and mental and behavioral disorders will have decreasing trends while diseases of the genitourinary system, diseases of the digestive system, diseases of the respiratory system, diseases of the nervous system, endocrine ,nutritional and metabolic diseases, diseases of blood and blood-forming organs and immune mechanisms, neoplasms, and infectious and parasitic diseases rates will have increasing trends.

Among these diseases, it is expected that unintentional injuries with decreasing rate of 54.19 per 100000 population during the years 2006 until 2035 will have the largest decline among the leading causes of death and diseases of the circulatory system with decreasing rate of 46.32 per 100000 population has the second rank.

Moreover, endocrine, nutritional and metabolic diseases with increasing rate of 187.52 and neoplasms with an increasing rate of 70.71 per 100000 population will have the largest increase in mortality rate from 2006 to 2035.

According to the ranking of the cause of death groups during the study years, it was predicted that diseases of the circulatory system are responsible for highest rate of mortality until 2031 in total population (first rank) while in 2035, endocrine, nutritional and metabolic diseases by rate of 197.71 per 100000 population will be responsible for the highest rate of mortality in Iran. In addition, it is predicted that from the year 2021 onwards, mental and behavioral disorders will be responsible for the lowest rate of mortality among the causes of death groups.

As can be seen in Table 3, it is predicted in the year 2035 that pattern of causes of death in Iran will drastically

Table 1. Crude death rate (per 100 thousand population) separated by age groups during the years 2006 to 2035

\begin{tabular}{|c|c|c|c|c|c|c|c|}
\hline \multirow{2}{*}{$\begin{array}{l}\text { Age Group } \\
\text { (years) }\end{array}$} & \multicolumn{7}{|c|}{ Year } \\
\hline & 2006 & 2011 & 2016 & 2021 & 2026 & 2031 & 2035 \\
\hline Under 5 & 453.63 & 323.5 & 225.86 & 182.79 & 147.93 & 119.72 & 101.08 \\
\hline $5-9$ & 41.42 & 40.71 & 27.34 & 23.31 & 19.87 & 16.94 & 14.91 \\
\hline $10-14$ & 39.9 & 38.57 & 36.48 & 35.32 & 34.19 & 33.1 & 32.25 \\
\hline $15-19$ & 88.68 & 87.21 & 72.57 & 68.38 & 64.43 & 60.71 & 57.89 \\
\hline $20-24$ & 123.45 & 102.99 & 74.02 & 62.95 & 53.54 & 45.53 & 40 \\
\hline $25-29$ & 136.14 & 107.18 & 67.63 & 54.97 & 44.68 & 36.32 & 30.77 \\
\hline $30-34$ & 142.64 & 126.51 & 80.33 & 67.42 & 56.58 & 47.49 & 41.28 \\
\hline $35-39$ & 157.44 & 142.14 & 103.3 & 90.18 & 78.72 & 68.71 & 61.63 \\
\hline $40-44$ & 199.5 & 186.31 & 158.88 & 148.59 & 138.96 & 129.95 & 123.17 \\
\hline $45-49$ & 313.02 & 260.22 & 193.74 & 168.22 & 146.05 & 126.81 & 113.25 \\
\hline $50-54$ & 471.86 & 446.87 & 352.79 & 322.9 & 295.54 & 270.49 & 252 \\
\hline $55-59$ & 725.53 & 680.87 & 562.76 & 519.84 & 480.19 & 443.57 & 416.29 \\
\hline $60-64$ & 1103.05 & 1128.82 & 997.5 & 958.23 & 920.5 & 884.25 & 856.28 \\
\hline $65-69$ & 1858.87 & 1717.25 & 1583.14 & 1520.56 & 1460.46 & 1402.74 & 1358.21 \\
\hline $70-74$ & 3173.36 & 3237.09 & 2408.59 & 2162.29 & 1941.18 & 1742.68 & 1598.6 \\
\hline $75-79$ & 5946.51 & 5660.44 & 5892.52 & 5990.22 & 6089.55 & 6190.51 & 6272.49 \\
\hline $80+$ & 11161.08 & 15318.19 & 19047.01 & 21754.18 & 24846.13 & 28377.54 & 31560.76 \\
\hline Total & 435.86 & 451.1 & 427.47 & 421.44 & 415.5 & 409.64 & 405.01 \\
\hline
\end{tabular}


Table 2. Mortality rate (per 100K population) of main disease groups from 2006 to 2035

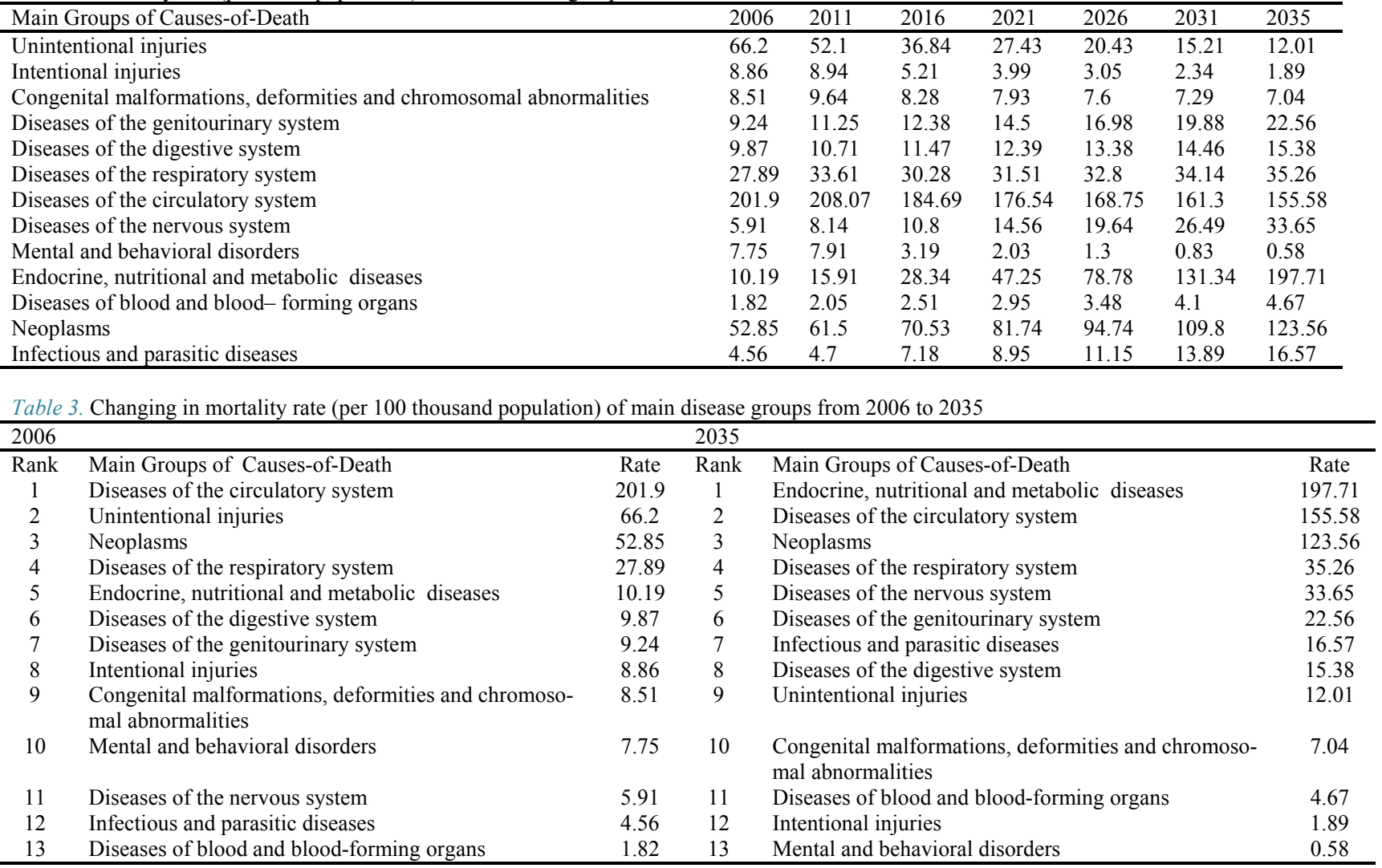

change when compared to the year 2006. So that in 2006, the diseases of the circulatory systems with the rate of 201.9 per 100000 population had the first rank of mortality while in 2035 , this disease will be placed in the second rank with 155.58 rate and endocrine, nutritional and metabolic diseases will shift from fifth to first rank of causes of death. It is also anticipated that unintentional injuries from second place in 2006 will decline to ninth place and diseases of blood and blood-forming organs and immune mechanisms, which were responsible for the lowest rate of mortality in 2006 will attain the eleventh rank in 2035 .

\section{DISCCUTION}

Since 2006, Iran's demographic architecture has entered into a demographic window which implies that almost $70 \%$ of the population of a country is placed at ages 15-64 and this trend lasts for nearly four decades $(14,15)$. Because of the changes in the structure of the population during the years of the survey, some changes are expected in the crude death rate and the pattern of causes of death.

According to the results of this study, it is anticipated that the crude death rate of Iran will have a declining trend in the total population. Based on the calculation of the World Bank, Iran's crude death rate has changed from 5.14 in 2006 to 4.54 in 2015 (16). Moreover, according to the report of World Development Indicators, Iran's crude death rate in 2006 and in 2015 was reported as 5.14 and 4.55 , respectively (17) where the declining pattern of the crude death rate in Iran is evident.

In anticipation of the main groups of cause-of-death in the Islamic Republic of Iran until the year 2035, endo- crine, nutritional and metabolic diseases with $32 \%$, diseases of the circulatory system with $25 \%$ and neoplasms with $20 \%$ will be the three major causes of death in the country.

In a project carried out by World Health Organization to estimate the number of causes of death in 2030, it is anticipated that diseases of the circulatory systems, neoplasms, diseases of the respiratory system and infectious and parasitic diseases will be the four major causes of death in the world in 2030 and diabetes will be responsible for $3.5 \%$ of all death. Also based on zoning of Millennium Development Goals (MDGs) has placed Iran in the region of South Asia, anticipates the first five causes of death in countries of the region in 2030 will include ischemic heart disease, chronic obstructive pulmonary disease, diarrheal disease, and lower respiratory infections. It is indisputable that the diseases of the circulatory systems and neoplasms are the leading causes of death in all countries (18). Nevertheless, based on the results of this study in Iran, diseases of the circulatory systems with a gentle slope will be on the decline and neoplasms will have an increasing trend. However, the highest rate of increase would be associated with the endocrine, nutritional and metabolic disease. The overall pattern of outbreaks endocrine, nutritional and metabolic disease in the world demonstrated that the incidence of these diseases has considerable growth in developing countries (19). However, increasing age leads to higher prevalence of diabetes, such that the prevalence of diabetes from $4.1 \%$ in those aged 25-44 years was more than $10 \%$ in those aged over 65 years old (20). Considering the change in age structure of 
Iran's population in 2035 and due to $21.4 \%$ increase in population aged over 45 years in comparison to 2015 (15), we expected a high prevalence of the disease and increase in mortality rate caused by this disease. In a country like Korea with growing elderly population, it has been estimated that cancers, cardiovascular disease, respiratory diseases, and diabetes, will be the most common causes of mortality in this country in 2030 (21).

\section{Conclusion}

Iran, in the near future, is faced with the aging population and high prevalence of non-communicable diseases; with continuing the current trends in resource allocation, many people's needs will remain unanswered. Thus, we should look beyond the health system and should promote public health using a comprehensive- preventative approach and applying suitable measures and policies prohibit the burden of population aging and noncommunicable diseases.

\section{Acknowledgments}

The researchers of this study are thankful to the death registration authorities at medical universities who have contributed to the data recordings.

\section{Conflict of interests}

The authors declare that they have no competing interests.

\section{References}

1. Greenberg RS, Daniels SR, Flanders WD, Eley JW, Boring JR. Medical Epidemiology. 4nd ed. New York: McGraw-Hill; 2001.pp.14-35.

2. World Health Organization. International statistical classification of diseases and related health problems. 10nd ed. Geneva; 1992.pp.87125 .

3. Naghavi M, Jafari N. Report of death in 4 province. Tehran: Ministry of Health and Medical Education; 1999.pp.25-45.

4. Stoto MA, Durch JS. Forecasting survival health and disability: Report on a workshop. Population and Development Review; 1993 Sep. Report No: 100322

5. Pollard J H. Projection of age-specific mortality rates. Population Bulletin of the United Nations. 1987;21(22):55-69.

6. Lee R D, Carter LR. Modelling and forecast US mortality. J Am Stat Assoc. 1992;87(419):659-671.

7. Di Cesare M, Murphy M. Forecasting mortality different approaches for different cause of death? The case of lung cancer; influenza, pneumonia, and bronchitis; and motor vehicle accident. Br Actuar J 2009;15(S1):185-211.

8. Deaton A, Pakson CP. Mortality, income, and income inequality over time in the Britain and the United States. Technical report. National Bureau of Economic Research Cambridge; 2001 May. Report No: c10354.

9. Komijani A, Koosheshi M, Niakan L. Iran mortality rates using LeeCarter model: Estimation and forecasting. Insur J. 2013;28 (4) :1- 25.

10. World Health Organization. Analyzing Mortality level and Cause of Death Based on WHO Standard Guideline. Geneva; 2013.pp.55-80.

11. Statistical Center of Iran. Census of Population and Housing 2006 2011[homepage on the Internet].Tehran: Statistical Center of Iran; [Updated 2012 Dec 16; cited 2015 Apr 2]. Available from: https://www.amar.org.ir.

12. Booth H, Hyndman R.J, Tickle, L, De Jong P. Lee-Carter mortality forecasting: A multi-country comparison of variants and extensions. Demograph Res J. 2006;15(9):289-310.

13. Lee RD. The Lee-Carter method for forecasting mortality, with various extensions and applications. North Am Actuari J. 2000;1(4):80-91.

14. Saraei H. The crowd valve Iran. Plan soc welfare developt J.
2010;1(1):33-45.

15. United Nations, Department of Economic and Social Affairs Population Division.World Population Prospects 2015 [homepage on the Internet]. New York: United Nations; [Updated 2015 Jun 10; cited 2016 Dec 20]. Available from: https://populationpyramid.net/world/.

16. The World Bank. Death rate, crude (per 1,000 people)[homepage on the Internet].Washington, DC: World Bank; [Updated 2016 Sep 5; cited 2017 Oct 20]. Available from: https://data.worldbank.org/indicator/ SP.DYN.CDRT.IN?locations=IR

17. Knoema.World Development Indicators [homepage on the Internet].Washington, DC: Knoema;[Updated 2016 Oct 10; cited 2017 Sep 15]. Available from: https://knoema.com/WBWDIGDF2017Sep/ world-development-indicators-wdi?tsId $=1608370$

18. World Health Organization. Projections of mortality and causes of death, 2015 and 2030. [homepage on the Internet].Geneva:World Health Organization; [Updated 2016 Jun 3; cited 2016 Oct 20]. Available from: http://www.who.int/healthinfo/global_burden_disease/ projections/en/.

19. Karronen M, Tuomilehto J, Libman I, Laprote R. A review of the recent epidemiological data on the worldwide incidence of type1 Diabetes mellitus.World Health Organization DIAMOND Project Group. Diabetologia 1994;38(6):642.

20. Institute of Endocrinology and Metabolism. Diabetes education for the elderly. Tehran; 2010. pp.23-67.

21. Yun JW, Son M. Forecasting Cause-Specific Mortality in Korea up to year 2032. J Korean Med Sci. 2016;31(6):892-901. 